\title{
A J2EE-based MIS design and implementation of light industry
}

\author{
Pan yong \\ Shanghai University of Engineering Science \\ Shanghai, China \\ ypan1998@163.com
}

\begin{abstract}
Based on J2EE architecture design of the three B/S mode of Light (beer) industry MIS system. Its functional modules include sales management, purchasing management, and quality management. Connect to the database using EJB components, to achieve a distributed concept, to bring high economic efficiency.
\end{abstract}

Keywords-MIS; J2EE; EJB; B / S

\section{INTRODUCTION}

With the development of computer communication network technology and e-commerce is widely used, the important role of enterprise information technology has been greatly improved. Through the construction of ERP systems can improve the level of enterprise information management and to improve the operating efficiency of enterprises, to provide scientific decision support for enterprise production.

\section{PARTICULARITY FOR BEER INDUSTRY INFORMATION NEEDS}

Diversity and uncertainty of product formulation, such that fluctuation of the manufacturing process is large, the formula needs:

- Diversity and uncertainty of product formulation, such that fluctuation of the manufacturing process is large, the formula needs .

- Costing complexity, some products require concentration or purity converted into a standard to calculate the cost of production and standards; some need to consider co-products, by-products.

- Equipment management should be done to ensure the stable operation of the equipment and production lines and production at full.

- Depth distribution model has become a trend in the beer business tool, flat management channel is channel development.

- Competitive analysis has become an important part of the market to work, to keep abreast of the main competitors of market dynamics, market activity for the enterprise market strategy to provide accurate basis.
- Frequent turnover of packaging has become a bottleneck in business management, information technology must be accompanied by a deposit of the sales process management, loan management bottle container, pour container management.

- Price policy, discount policy flexibility, diversity has become a competitive asset.

- Lot of difficulty offsite warehouse has become unified management

\section{SYSTEM ARCHITECTURE DESIGNE}

According to the distributed multi-tier application model J2EE specification defines the nature and components will be employed in different applications to different types of application servers. System will be based on the difference between the various components of the information system on the functional use of the entire multi-tier architecture application system is divided into three separate layers, application software will construct on this framework, they are client tier (Client tier), The logical layer, presentation logic layer (Presentation logic tier) and business logic layer (Business logic tier), the data layer (Data tier). Application layer are disposed in respective different types of application servers, have good stretching ability.

\section{1) Client layer}

Information systems for users to interact, receive user input and user input is analyzed to check and make the appropriate treatment. Also can display the high-level server according to specific business rules to run after the result of the calculation, the client layer is usually composed by the client process, these processes dynamically created and destroyed by the browser. WEB-based client application, the user starts the client browser, download WEB layer static or dynamic HTML pages generated by the JSP or Servlets HTML pages from WEB server.

\section{2) Logical layer}

It contains the presentation logic and business logic layer consists of: performance logic layer (WEB layer): means for receiving a request to the business logic layer and coming from the browser to process the request, but the request processing results sent to the browser. 
These processes mainly by the JSP page, WEB-based Java Applets and Servlets used to dynamically generate HTML pages constituted. These basic elements in the assembly process to create the WEB by packing components. WEB layer runs WEB component depends WEB container to support such a response to customer requests and inquiries EJB components and other functions. By WEB server, authentication server, performance management, the use of the serial method of physical realization. WEB network server using Tomcat5.0.

Business logic layer: Responsible for the performance of client requests coming logical layer and business rules in accordance with its business processes. While completing the request result to the presentation logic layer, if desired, the processing results need to be stored to the data layer and logical checks, information retrieval, and other functions. This layer is the most critical part of whole system. Including the monitoring process, handling process and other processes and database

operations. Deploy and run the business logic layer of the EJB component, depending on the EJB container to manage such affairs, life cycle, state transition, multi-threading and resource storage.

\section{3) Data Layer}

Primarily for the business logic layer to provide data services, responsible for storing persistent data. Such as storage logical layer results, the data returned search results and other business logic layer. The basic application data can be expanded. Structure of the system can be accessed using the metadata application data system management and maintenance. If you already have a data structure has changed, just change its data structure by the data dictionary maintenance tools, maintenance tools and then maintain its associated application by application logic.

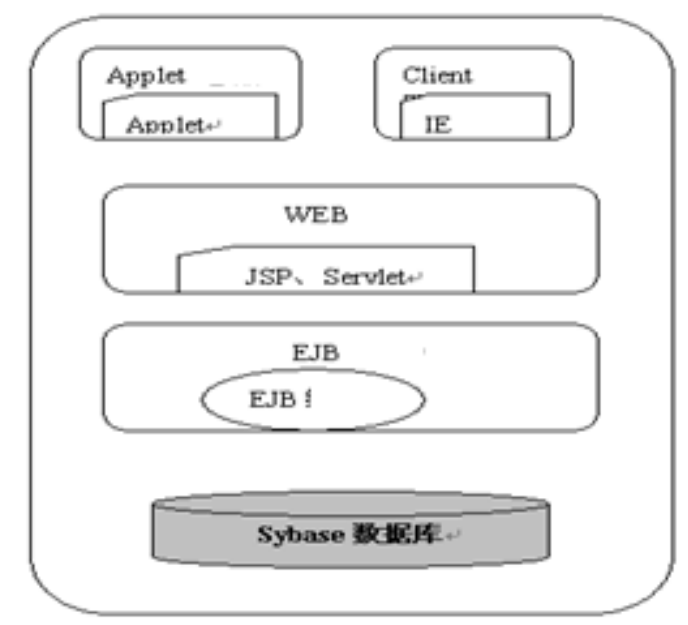

Fig. 1. systemarchitecture

\section{SYSTEM IMPLEMENTATION}

The system consists of basic data, purchasing management, inventory management, sales management, quality management, integrated, query subsystem, five production and management of distributed databases (four business databases distributed in four plants, a business database at headquarters ) and one as the head office to query the database query with the composition. Its network topology is as shown below.

The relationship between the various subsystems of the "public relations environment coupling", there is no relationship between calling each other, they were associated through a shared database. Application development platform: server operating system options with Solaris, the client operating system used Windows operating system, browser choice IE browser, the database system used ASE12 Enterprise Edition, Web server selection Apache, the choice of development tools, data released Jbuilder 10 enterprise Edition.

The key lies in the system software development, database development and data release. The main database with powerful ASE DBMS is functionality to support their realization. In the implementation process of the middle layer of business requirements analysis and based on actual business needs, user-friendly principles of humanity divided blocks of a program, and according to the object-oriented analysis and design methods to associate among several modules, making it become a powerful overall system to meet the needs of everyday business. System is designed to function as independent but interrelated modules:

Each subsystem functions are summarized below:

- basic data management subsystem provides management of common data needed for each subsystem.

- sales management subsystem provides sales invoice processing, packaging, deposit management, product shipment management, product management and sales out of storage for statistical analysis.

- procurement management subsystem provides MRP declaration and approval, procurement planning, various statistical analysis.

- inventory management subsystem materials out of storage management, inquiry management of materials in the library and various systems

- Quality Management Subsystem inspection data by a variety of quality inspection process system, the system according to process. 
- requirements of the process indicators data, providing quality, process analysis of a variety of ways to generate a variety of system reports.

- Integrated Query subsystem factory production and management information collected to query the database at headquarters, offers a variety of query and analysis methods for leadership decision-making.

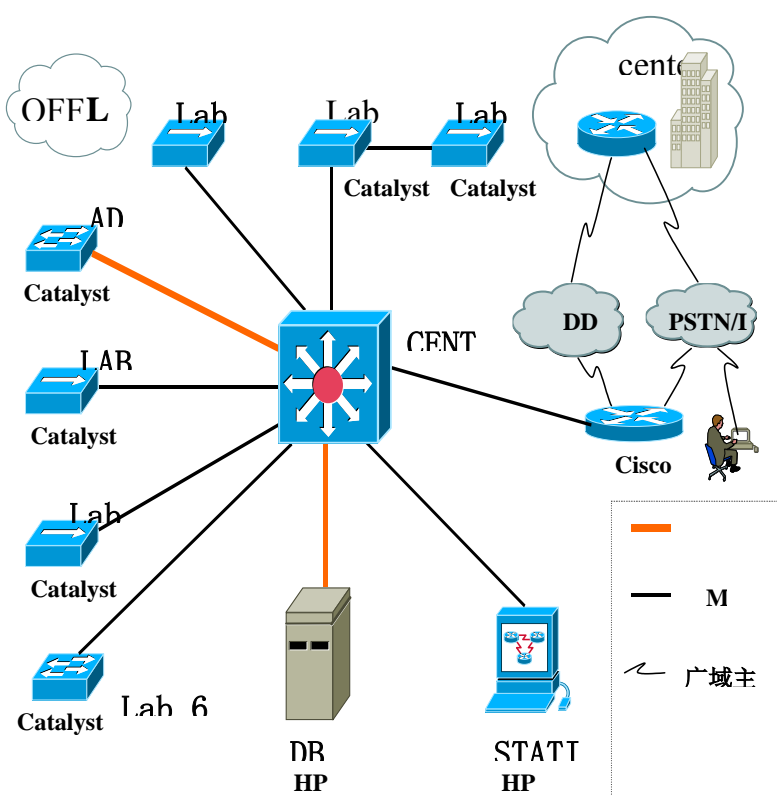

System

Fig 2 System architeture

\section{SYSTEM FEATURES}

Database structure designed to meet the integrity and security of data, models of dynamic relationship with a third normal form, the pattern of a special relationship with at least a first normal form, the logic level to efficiently support the operational requirements of the user's data and has high utilization of storage space for the final design goals.

- object-oriented analysis and design techniques;

- distributed multi-tier architecture technology;

- using J2EE technology system:

- develop the entire system based on object-oriented technology, components for flexible forms of organization. Open system architecture, and strive to achieve several restructuring application system to meet different business needs.

- combination of advanced and practical, both consider the needs of the future development of corporate strategic objectives, but also take into account the needs of enterprises of all types of business currently.

\section{CONCLUSION}

According to Beer industry enterprise status, the use of advanced computer processing means, the introduction of advanced management concepts related industry management system, breaking the traditional single in order to achieve some of the features and the development of the concept of software for the purpose of the enterprise from technology and management the dual perspective to measure the actual needs of enterprises, so as to reduce the large number of enterprise resources, human and financial resources, the enterprise has brought great benefits.

\section{References}

[1] Gunasekaran A,MARRI H B,MCGAUGHEY R E. E-commerce and its impact on operations management[J].International Journal of Production Economics,2002.185-197.LEVIS K. Electronic Commerce [J].British Telecommunications Engineering, 1996,(04):281-285.

[2] I.S. Jacobs and C.P. Bean, "Fine particles, thin films and exchange anisotropy," in Magnetism, vol. III, G.T. Rado and H. Suhl, Eds. New York: Academic, 1963, pp. 271-350.

[3] Susan M,MUDARNBI,RAJ AGGARWAL. Industrial distributors Can they survive in the new economy?[J].Industrial Marketing Management,2002,(31):1-9. R. Nicole, "Title of paper with only first word capitalized," J. Name Stand. Abbrev., in press.

[4] Yan(Jenny)Liu,Ian Gorton,Anna Liu,Shiping Chen. Evaluating the scalability of enterprise javaBeans technology[A].NW Washington,DC USA:IEEE Computer Society,2002.74-83.

[5] Shaofeng Wang, Kehong Wang. Research of JavaEJB[EB/OL]. http://teamsun com.cn/usseer/ejb.htm.

[6] EJB 2.0[EB/OL].http://www.umlchina.com/CBD/Newindex1.htm,.. 